\title{
Angiopoietin-like protein 2 sensitively responds to weight reduction induced by lifestyle intervention on overweight Japanese men
}

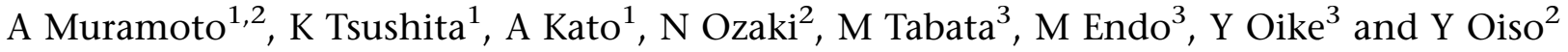 \\ ${ }^{1}$ Division of Health Development of Comprehensive Health Science Center, Aichi Health Promotion Foundation, Aichi-ken, \\ Japan; ${ }^{2}$ Department of Endocrinology and Diabetes, Nagoya University Graduate School of Medicine, Nagoya, Japan and \\ ${ }^{3}$ Department of Molecular Genetics, Graduate School of Medical Sciences, Kumamoto University, Kumamoto, Japan
}

Objective: Overexpression of Angiopoietin-like protein 2 (Angptl2) in obese adipose tissues promotes adipose tissue inflammation and its-related metabolic abnormalities. In a comparative study with adiponectin, we investigated whether alterations in serum Angptl2 concentrations reflect the effect of lifestyle intervention on weight loss and improved metabolic parameters in overweight subjects.

Methods: A total of 154 Japanese men (age, $40.9 \pm 5.1$ years; body mass index, $26.9 \pm 3.6 \mathrm{~kg} \mathrm{~m}^{-2}$; abdominal circumference, $94.1 \pm 8.9 \mathrm{~cm}$ ) underwent a 3-month lifestyle intervention and underwent follow-up for 3 months thereafter.

Results: Decreased serum Angptl2 levels, but not increased serum adiponectin levels, were immediately apparent at the end of 3-month lifestyle intervention. Angptl2 levels continued to decrease for 3 months in parallel with body weight loss and improvement in metabolic indicators. In subjects showing $\geqslant 6 \%$ weight reduction, markedly reduced Angptl 2 levels were detected at the end of 3-month intervention, whereas increased adiponectin levels were detected 3 months after the end of intervention. Multivariate analysis revealed changes in serum Angptl2 levels associated with changes in triglycerides (TGs), aspartate aminotransferase and alanine aminotransferase. In contrast, changes in serum adiponectin levels were associated with altered high-density lipoprotein cholesterol (HDL-C) and fasting plasma glucose levels.

Conclusion: A 3-month lifestyle intervention promoted weight reduction and improved glucose and lipid metabolism, an effect maintained 3 months later. Notably, our findings indicate that decreased Angptl 2 levels are a good indicator of reduced visceral fat and metabolic improvement at early stages of lifestyle intervention. Thus, Angptl2 reflects adiposity and might be a key protein to regulate inflammation and TG metabolism, whereas adiponectin levels could reflect improved glucose and HDL-C metabolism.

Nutrition and Diabetes (2011) 1, e20; doi:10.1038/nutd.2011.16; published online 7 November 2011

Keywords: metabolic syndrome; lifestyle intervention; body weight reduction; angiopoietin-like protein 2; adiponectin

\section{Introduction}

Obesity is a pandemic medical and social problem that increases lifestyle-related diseases, such as cardiovascular disease, type 2 diabetes, hypertension, dyslipidemia and cancer, all of which result in increased mortality. ${ }^{1-5}$ Therefore, antagonizing weight gain is critical to decrease

Correspondence: $\mathrm{Dr} \mathrm{K}$ Tsushita, Division of Health Development of Comprehensive Health Science Center, Aichi Health Promotion Foundation, 1-1 Gengoyama, Morioka, Higashiura-cho, 470-2101 Aichi, Japan.

E-mail: k-tsushita@grp.ahv.pref.aichi.jp

or Dr Y Oike, Department of Molecular Genetics, Graduate School of Medical Sciences, Kumamoto University, 1-1-1 Honjo, Kumamoto 860-8556, Japan. E-mail: oike@gpo.kumamoto-u.ac.jp

Received 2 September 2011; accepted 21 September 2011 occurrence of these diseases. Recent reports demonstrate that weight loss has ameliorating effects on type 2 diabetes, hypertension or dyslipidemia, independent of differences in race, sex, age, intervention method and intervention period. ${ }^{6-9}$

Recently, the concept has emerged that obesity-related inflammation is associated with high risk of type 2 diabetes and cardiovascular diseases. ${ }^{10-15}$ Circulating levels of C-reactive protein (CRP), fibrinogen and some adipose tissue-derived cytokines, all associated with inflammation, are decreased by body weight reduction, an occurrence associated with improved insulin resistance. ${ }^{6,16-19}$

More recently, we revealed that circulating levels of angiopoietin-like protein 2 (Angptl2), which is a stress responsive adipose tissues-secreted protein, were higher than 
normal in cases of obesity in human and mice, particularly in cases with visceral fat accumulation, leading to chronic adipose tissue inflammation and subsequent development or progression of insulin resistance and metabolic syndrome. ${ }^{20-22}$ We observed accumulation of fat in the liver and skeletal muscle was mild in Angptl2 knockout mice compared with wild-type mice, and Angptl2 deletion ameliorated adipose tissue inflammation. ${ }^{20}$ We also observed significant decreases in circulating Angptl2 concentrations in obese diabetic men following treatment with the PPAR $\gamma$ agonist pioglitazone, and the percent decrease in Angptl2 levels was positively correlated with the percent decreases in visceral fat area. These findings suggest that visceral fat is a likely primary source of circulating Angptl2 and that levels of that factor are significantly correlated with systemic insulin resistance and inflammation. ${ }^{20-22}$

But it remains unknown whether Angptl2 could respond to weight reduction and its-related metabolic abnormalities by lifestyle intervention, and if so, we'd like to know the difference between angptl2 and adiponectin.

Thus, the aim of the present study was to investigate whether Angptl2 levels reflect weight reduction, the degree of weight reduction and obesity-related metabolic abnormalities. For comparison, we monitored the influence of weight reduction on adiponectin levels, which reportedly increase in the circulation with weight loss and serve as a biomarker to assess the improvement of obesity and its-related metabolic abnormalities. ${ }^{6-9}$

\section{Materials and methods}

\section{Subjects}

'Overweight subjects' were recruited to participate in a lifestyle intervention program through newspaper or website advertising. We defined the subjects with a body mass index $(\mathrm{BMI}) \geqslant 25.0 \mathrm{~kg} \mathrm{~m}^{-2}$ or an abdominal circumference $\geqslant 85 \mathrm{~cm}$ as 'overweight subject' in this study, because this criteria is established as an adequate risk for categorizing 'obesity disease' in Japan in relation to obesity-related complications in Japan. ${ }^{23}$ All subjects gave written consent after having received verbal and written information about this study. The Ethical Review Board of the Aichi Health Plaza Comprehensive Health Science Center approved the study procedures.

\section{Study design}

Subjects underwent lifestyle intervention for 3 months and then were observed without intervention for 3 months thereafter. At the beginning of intervention, and at the 3- and 6-month time points, a questionnaire about lifestyle, anthropometric measurements, blood pressure (BP) measurements and blood tests were performed. The questionnaire contains smoking status, drinking habit and exercise habit. For anthropometric measurements, height, weight, abdominal circumference and body fat percentage (\% fat) were measured. For blood tests, triglyceride (TG), high-density lipoprotein cholesterol (HDL-C), low-density lipoprotein cholesterol (LDL-C), fasting plasma glucose (FPG), hemoglobin A1c (HbA1c), serum insulin (insulin), aspartate aminotransferase (AST), alanine aminotransferase (ALT), high-sensitive CRP (hs-CRP), Angptl2 and adiponectin levels were measured.

\section{Lifestyle intervention}

Subjects received detailed results of an examination that they underwent at the beginning of intervention and attended a lecture regarding the association of obesity and health problems and the benefits of weight reduction. That lecture included illustrations and graphs depicting the relationship of lifestyle on metabolic syndrome-related conditions. Subjects were then advised by support staff (including a public health nurse, nutritionist and health exercise trainer) to set their own behavioral targets. Then, for 3 months, subjects received lifestyle improvement support for the purpose of weight reduction. Actually, subjects were instructed in proper meal preparation for the energy-balance or received support in the form of exercise training. We instructed them by interview or supported them by e-mail once or twice a month, depending on subjects' living conditions.

\section{Anthropometric measurements}

Weight was measured with the subjects wearing light clothing and barefoot. BMI was calculated as weight $(\mathrm{kg})$ divided by height $\left(\mathrm{m}^{-2}\right)$. Body fat percentage was determined using the bioelectrical impedance analysis (BIA) method (TBF-102; Tanita Corporation, Tokyo, Japan). Abdominal circumference was measured at the level of the umbilicus (horizontal to the ground). Systolic and diastolic blood pressure was measured using an automatic sphygmomanometer (SunTech Medical, Morrisville, NC, USA).

\section{Biochemical analysis}

Blood samples were taken after overnight fasting around at 0900 hours. LDL-C was measured using the direct method (Determiner L, LDL-C, Kyowa Medex Co., Ltd, Tokyo, Japan). HbA1c ( Japan Diabetes Society) was measured using the latex agglutination method with a commercial test kit (Kyowa Medex Co., Ltd, Tokyo, Japan). The HbA1c defined by the National Glycohemoglobin Standardization Program, which is the internationally used HbA1c, is expressed by adding $0.4 \%$ to the HbA1c (JDS). ${ }^{24}$ The HbA1c data are shown by HbA1c (National Glycohemoglobin Standardization Program). Insulin was measured using an enzyme immunoassay with a commercial test kit (Eiken Chemical Co, Ltd, Tokyo, Japan). Homeostasis model assessment of the insulin resistance index (HOMA-IR) was calculated using a 
method described elsewhere. ${ }^{25}$ There is a good correlation between HOMA-IR and glucose infusion rate obtained by the euglycemic-hyperinsulinemic clamp method. ${ }^{26}$ Given the combination of accuracy and ease of testing, we use HOMA-IR as an index of insulin resistance. Hs-CRP levels were measured using the latex agglutination method with a commercial test kit (N-assay LA, CRP-T, Nittobo, Tokyo, Japan). Adiponectin was measured by ELISA using a test kit (Adiponectin ELISA kit; Otsuka Pharmaceutical Co., Ltd, Tokyo, Japan).

Angptl2 was measured by ELISA as previously reported. $^{20,27}$ In brief, the K2-1A1 mouse monoclonal antibody was fixed to 96-well plates. After 10-fold dilution, serum samples were immobilized on plates for $1 \mathrm{~h}$ at $37^{\circ} \mathrm{C}$, followed by washing with PBS containing $0.05 \%$ Tween 20 (PBST) and addition of horseradish peroxidase-conjugated K1-12A4 mouse monoclonal antibody. After $1 \mathrm{~h}$ of incubation at $4{ }^{\circ} \mathrm{C}$, plates were washed with PBS containing $0.05 \%$ Tween 20 , and a tetramethylbenzidine detection reagent was added to the wells. After $30 \mathrm{~min}$, the reaction was stopped by addition of an equal amount of $1 \mathrm{~N} \mathrm{H}_{2} \mathrm{SO}_{4}$, and absorbance was measured at $450 \mathrm{~nm}$. We confirmed the validity of this ELISA system through the following experiments. Absorbance at $450 \mathrm{~nm}$ increased linearly with human Angptl2 calibrators of 50-350 pg per assay with the least detectable concentration of $50 \mathrm{pg}$ (correlation coefficient $>0.99$ ). In all three intra-assay determinations of the same samples showed coefficients of variation less than $5 \%$ at all Angptl2 concentrations tested. The mean \pm s.d. concentrations measured (and coefficient of variation) were: sample 1, 1.76 \pm $0.05 \mathrm{ng} \mathrm{ml}^{-1}$ (2.9\%); sample 2, $0.43 \pm 0.01 \mathrm{ng} \mathrm{ml}^{-1}$ (3.2\%); and sample $3,0.14 \pm 0.01 \mathrm{ng} \mathrm{ml}^{-1}(3.3 \%)$. The three inter-assay determinations of the same serum gave coefficients of variation of less than 10\%: sample $1,1.82 \pm 0.09 \mathrm{ng} \mathrm{ml}^{-1}$ $(5.2 \%)$; sample $2,0.44 \pm 0.02 \mathrm{ng} \mathrm{ml}^{-1}$ (4.6\%); and sample 3 , $0.142 \pm 0.01 \mathrm{ng} \mathrm{ml}^{-1}(5.0 \%)$. Plasma concentrations of other factors were determined using standard clinical biochemistry methods.

\section{Statistical analysis}

To assess the effectiveness of lifestyle intervention, changes in population characteristics at the beginning (0), end of the 3 -month intervention or end of the 6-month program (3-month intervention plus 3-month observation) were assessed with the Wilcoxon signed rank test.

We used partial correlation analysis to examine association between baseline Angptl2 or adiponectin levels and baseline values of each laboratory measurement. We also analyzed the association between changes in Angptl2 or adiponectin levels and changes in each laboratory parameter, from the beginning of the intervention to 3 and then 6 months later. We tested baseline data and changes for normal distribution by Kolmogorov-Smirnov method. From this result, we logarithmically transformed TG, IRI, HOMA-IR, AST, ALT, hs-CRP at baseline and changes in hs-CRP at 3 and 6 months.
Multiple linear regression analysis was conducted for each change in BP, lipid metabolism, glucose metabolism and liver function from the beginning of the intervention to 3 and 6 months, later as target variables, and values highly correlated with each variation as explanatory variables. Furthermore, subjects were grouped per $2 \%$ weight reduction at 3 or 6 months after the beginning of intervention. Changes in laboratory data values were analyzed by analysis of variance or the Kruskal-Wallis test (for changes in diastolic blood pressure and $\mathrm{HbA1c}$ after 3 months). Statistically significant changes were compared using multiple comparison by the Bonferroni method.

We used the statistics software PASW Statistics Base 18.0 (SPSS, Tokyo, Japan) and expressed results as means \pm s.d. or s.e.m. Differences at the level of $P<0.05$ were considered statistically significant.

\section{Results}

Baseline characteristics of subjects

A total of 154 men were enrolled. The average age was $40.9 \pm 5.1$ years and BMI was $26.9 \pm 3.6 \mathrm{~kg} \mathrm{~m}^{-2}$ (Table 1). Patients with an endocrine disorder or those undergoing drug treatment for diabetes, hypertension or dyslipidemia were excluded. Clinical and laboratory examination to characterize baseline parameters before lifestyle intervention revealed one patient with diabetes, which required therapy. During the 3-month lifestyle intervention, seven subjects dropped out of the study because of traffic accidents or occupational reasons. During the subsequent 3-month follow-up, 11 of the remaining 146 subjects could not be followed for various reasons, such as occupational issues or lack of motivation. Finally, 135 subjects who could be followed for all 6 months of the program were analyzed (continuation rate of $87.7 \%$ ) (Figure 1).

At the baseline, correlation analysis adjusted by age showed a significant positive correlation of Angptl2 levels with adiposity, as estimated by BMI, abdominal circumference and fat mass (Table 2a). In contrast, baseline adiponectin levels showed an inverse correlation with adiposity (Table $2 \mathrm{a}$ ).

Correlation analysis adjusted by age and BMI to compare baseline Angptl2 levels with clinical and laboratory data showed a significant positive correlation of Angptl2 with diastolic Blood Pressure, TG, Insulin and HOMA-IR and a significant inverse correlation of Angptl2 with HDL-C (Table 2b). By contrast, baseline adiponectin levels were inversely correlated with TG, Insulin, HOMA-IR, AST, ALT and hs-CRP, and showed a significant positive correlation with HDL-C. Baseline Angpt12 and adiponectin levels showed an inverse relationship trend $(P=0.056)$ (Table $2 b)$. When we added smoking status, drinking habit and exercise habit as control variables, association between Angptl2 and adiponectin at baseline was significant $(r=-0.215, P=0.014)$. 
Table 1 Characteristics of the 135 subjects at 0 (baseline), 3 and 6 months after initiation of the intervention

\begin{tabular}{|c|c|c|c|c|c|}
\hline & Baseline & 3 months & P-value & 6 months & P-value \\
\hline Age & $40.9 \pm 5.1$ & & & & \\
\hline Weight (kg) & $79.4 \pm 11.8$ & $77.0 \pm 11.5$ & $<0.001$ & $76.4 \pm 11.6$ & $<0.001$ \\
\hline BMI $\left(\mathrm{kg} \mathrm{m}^{-2}\right)$ & $26.9 \pm 3.6$ & $26.1 \pm 3.6$ & $<0.001$ & $25.9 \pm 3.6$ & $<0.001$ \\
\hline Abdominal circumference $(\mathrm{cm})$ & $94.1 \pm 8.9$ & $91.4 \pm 9.0$ & $<0.001$ & $90.5 \pm 9.5$ & $<0.001$ \\
\hline$\%$ fat (\%) & $26.6 \pm 5.4$ & $24.6 \pm 4.7$ & $<0.001$ & $24.4 \pm 4.8$ & $<0.001$ \\
\hline Fat mass $(\mathrm{kg})$ & $21.6 \pm 7.5$ & $19.4 \pm 6.7$ & $<0.001$ & $19.1 \pm 6.8$ & $<0.001$ \\
\hline SBP $(\mathrm{mm} \mathrm{Hg})$ & $121.6 \pm 12.4$ & $121.9 \pm 11.9$ & 0.523 & $121.7 \pm 12.3$ & 0.992 \\
\hline $\mathrm{DBP}(\mathrm{mm} \mathrm{Hg})$ & $72.4 \pm 10.3$ & $76.6 \pm 9.9$ & $<0.001$ & $75.4 \pm 10.6$ & $<0.001$ \\
\hline TG $(\mathrm{mg}$ per $100 \mathrm{ml})$ & $149.8 \pm 114.2$ & $122.6 \pm 71.7$ & $<0.001$ & $136.2 \pm 116.3$ & 0.009 \\
\hline HDL-C (mg per $100 \mathrm{ml})$ & $58.4 \pm 13.2$ & $55.5 \pm 12.1$ & $<0.001$ & $57.1 \pm 12.8$ & 0.051 \\
\hline LDL-C (mg per $100 \mathrm{ml}$ ) & $134.8 \pm 35.3$ & $125.1 \pm 31.0$ & $<0.001$ & $126.7 \pm 33.2$ & $<0.001$ \\
\hline LDL-C/HDL-C & $2.43 \pm 0.84$ & $2.39 \pm 0.86$ & 0.166 & $2.35 \pm 0.89$ & 0.155 \\
\hline FPG (mg per $100 \mathrm{ml}$ ) & $99.9 \pm 12.0$ & $96.3 \pm 9.7$ & $<0.001$ & $96.3 \pm 9.9$ & $<0.001$ \\
\hline HbA1c (\%) & $5.38 \pm 0.45$ & $5.20 \pm 0.36$ & $<0.001$ & $5.22 \pm 0.35$ & $<0.001$ \\
\hline Insulin ( $\mathrm{mcU}$ ml-1) & $10.12 \pm 9.17$ & $7.37 \pm 5.14$ & $<0.001$ & $8.09 \pm 5.74$ & $<0.001$ \\
\hline HOMA-IR & $2.57 \pm 2.71$ & $1.79 \pm 1.37$ & $<0.001$ & $1.96 \pm 1.49$ & $<0.001$ \\
\hline AST $\left(\mathrm{IUI}^{-1}\right)$ & $25.5 \pm 9.0$ & $22.2 \pm 8.5$ & $<0.001$ & $23.0 \pm 8.7$ & 0.005 \\
\hline $\operatorname{ALT}\left(\mathrm{IUI}^{-1}\right.$ & $37.1 \pm 21.9$ & $28.6 \pm 15.9$ & $<0.001$ & $30.0 \pm 20.2$ & $<0.001$ \\
\hline Hs-CRP (mg per $100 \mathrm{ml}$ ) & $0.086 \pm 0.156$ & $0.086 \pm 0.125$ & 0.907 & $0.090 \pm 0.120$ & 0.811 \\
\hline Angptl2 $\left(\mathrm{ng} \mathrm{ml}^{-1}\right)$ & $3.02 \pm 1.18$ & $2.79 \pm 1.11$ & 0.001 & $2.65 \pm 1.08$ & $<0.001$ \\
\hline Adiponectin $\left(\mathrm{mcg} \mathrm{ml}^{-1}\right)$ & $6.59 \pm 3.78$ & $6.22 \pm 3.94$ & $<0.001$ & $6.45 \pm 3.93$ & 0.108 \\
\hline
\end{tabular}

Abbreviations: Angpt12, angiopoietin-like protein 2; ALT, alanine aminotransferase; AST, aspartate aminotransferase; BMI, body mass index; DBP, diastolic blood pressure; FPG, fasting plasma glucose; HbA1c, hemoglobin A1c; HOMA-IR, homeostasis model assessment of the insulin resistance index; Hs-CRP, high sensitive C-reactive protein; HDL-C, high-density lipoprotein cholesterol; LDL-C, low-density lipoprotein cholesterol; SBP, systolic blood pressure; TG, triglyceride. Data are presented as means \pm s.d. Statistical differences of clinical and laboratory data at the 3-and 6-month time points compared with baseline values were examined using the Wilcoxon signed rank test.

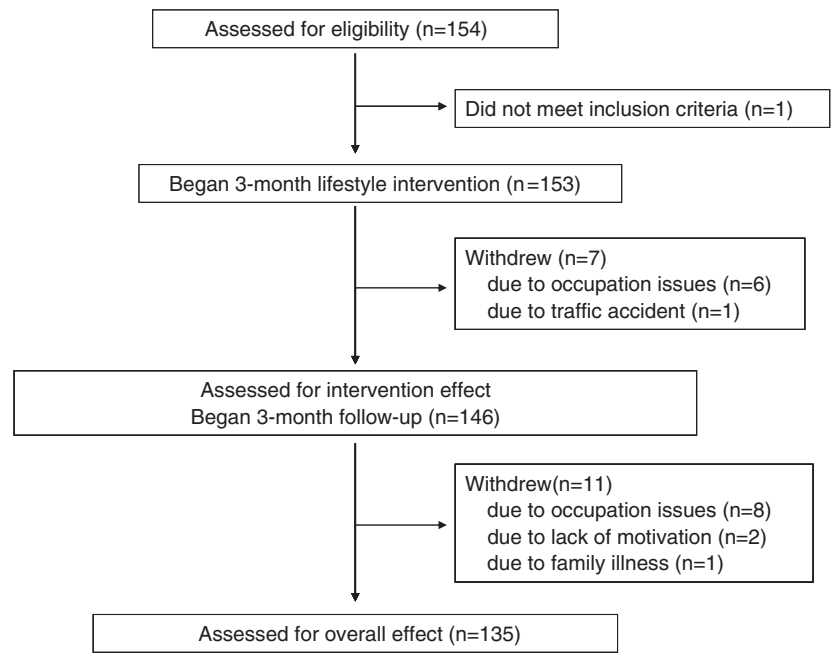

Figure 1 Flow of participants through the intervention and follow-up study.

Changes in anthropometric and biochemical parameters after 3 and 6 months

As shown in Table 1, subjects' weight at 3 and 6 months after the beginning of lifestyle intervention was decreased significantly compared with baseline values (reduced by $2.4 \pm 2$. $5 \mathrm{~kg}$ and $2.9 \pm 3.5 \mathrm{~kg}$, respectively). In addition, BMI, abdominal circumference, \% fat, fat mass, TG, LDL-C, FPG, HbA1c, insulin, HOMA-IR, AST and ALT levels were
Table 2a Correlations of Angptl2 and adiponectin levels with anthropometric measurements at baseline levels

\begin{tabular}{lcccccr}
\hline & \multicolumn{2}{c}{ Angpt/2 } & & \multicolumn{2}{c}{ Adiponectin } \\
\cline { 2 - 3 } \cline { 6 - 7 } & $\mathrm{r}$ & $\mathrm{P}$ & & $\mathrm{r}$ & $\mathrm{P}$ \\
\hline BMI & 0.257 & 0.003 & & -0.242 & 0.005 \\
Abdominal circumference & 0.262 & 0.002 & & -0.321 & $<0.001$ \\
Fat mass & 0.268 & 0.002 & & -0.277 & $<0.001$ \\
\hline
\end{tabular}

Abbreviations: Angpt|2, angiopoietin-like protein 2; BMI, body mass index. Analysis was performed and adjusted by age. $r$ and $P$ indicate correlation coefficients and $P$-values, respectively.

significantly reduced, indicating that the 3-month lifestyle intervention significantly reduced adiposity and ameliorated glucose and lipid metabolism. There was no significant change in hs-CRP levels at 3 and 6 months after the beginning of lifestyle intervention compared with those observed at baseline. Serum Angpt12 levels were significantly decreased from $3.02 \pm 1.18$ to $2.79 \pm 1.11 \mathrm{ng} \mathrm{ml}^{-1}$ (vs baseline value, $P=0.001$ ) 3 months after the beginning of intervention, and significantly decreased to $2.65 \pm 1.08 \mathrm{ng} \mathrm{m}^{-1}$ (vs baseline value, $P<0.001$ ) at the 6 -month time point. Although serum adiponectin levels were expected to increase based on previous reports, ${ }^{6-9}$ they decreased from $6.59 \pm 3.78 \mu \mathrm{g} \mathrm{ml}^{-1}$ to $6.22 \pm 3.94 \mu \mathrm{g} \mathrm{ml}^{-1}$ (vs baseline value, $P=0.001$ ) by the 3-month time point. At the 6-month time point, serum adiponectin levels were significantly increased 
compared with those at the 3-month time point $(P=0.006)$, however, they were not higher than baseline values (vs baseline value, $P=0.108$ ).

Table 2b Correlations of Angptl2 and adiponectin levels with blood pressure and laboratory data at baseline levels

\begin{tabular}{|c|c|c|c|c|}
\hline & \multicolumn{2}{|c|}{ Angpt/2 } & \multicolumn{2}{|c|}{ Adiponectin } \\
\hline & $r$ & $P$ & $r$ & $P$ \\
\hline SBP & 0.096 & 0.271 & -0.151 & 0.084 \\
\hline DBP & 0.247 & 0.004 & -0.149 & 0.087 \\
\hline $\log$ TG & 0.255 & 0.003 & -0.291 & 0.001 \\
\hline HDL-C & -0.424 & $<0.001$ & 0.299 & $<0.001$ \\
\hline LDL-C & 0.070 & 0.421 & -0.112 & 0.200 \\
\hline FPG & 0.085 & 0.331 & -0.069 & 0.432 \\
\hline $\mathrm{HbA1c}$ & 0.039 & 0.657 & -0.108 & 0.217 \\
\hline Log Insulin & 0.386 & $<0.001$ & -0.235 & 0.006 \\
\hline Log HOMA-IR & 0.368 & $<0.001$ & -0.232 & 0.007 \\
\hline $\log$ AST & 0.100 & 0.250 & -0.193 & 0.026 \\
\hline $\log$ ALT & 0.058 & 0.506 & -0.240 & 0.005 \\
\hline Log Hs-CRP & 0.093 & 0.287 & -0.278 & 0.001 \\
\hline Angptl2 & - & - & -0.166 & 0.056 \\
\hline Adiponectin & -0.166 & 0.056 & - & - \\
\hline
\end{tabular}

Abbreviations: Angptl2, angiopoietin-like protein 2; ALT, alanine aminotransferase; AST, aspartate aminotransferase; DBP, diastolic blood pressure; FPG, fasting plasma glucose; HbA1c, hemoglobin A1c; HOMA-IR, homeostasis model assessment of the insulin resistance index; Hs-CRP, high sensitive C-reactive protein; HDL-C, high-density lipoprotein cholesterol; LDL-C, low-density lipoprotein cholesterol; SBP, systolic blood pressure; TG, triglyceride. Analysis was performed and adjusted by age and BMI. $r$ and $P$ indicate correlation coefficients and $P$-values, respectively.
Changes in serum Angptl2 levels showed a significant positive correlation with changes in BMI, abdominal circumference and fat mass immediately after the intervention, whereas those in serum adiponectin levels showed an inverse correlation with BMI and fat mass values, but not with abdominal circumference (Table 3). By the 6-month time point, changes in serum Angptl2 levels continued to show a significant positive correlation with BMI, abdominal circumference and fat mass values, whereas serum adiponectin levels were inversely correlated with these parameters (Table 3).

Regarding changes in clinical parameters, decreases in serum Angptl2 levels showed a significant positive correlation with TG, AST, ALT and hs-CRP immediately after the intervention, whereas those in serum adiponectin levels showed a positive correlation with HDL-C and LDL-C. At the end of the program, changes in serum Angptl2 levels showed a significant positive correlation with TG, AST and hs-CRP values, and an inverse correlation with HDL-C, whereas serum adiponectin levels showed a positive correlation with HDL-C values and an inverse correlation with FPG (Table 3). Notably, we observed an inverse correlation between Angptl 2 and adiponectin levels after 6 months, but not after 3 months $(r=-0.186, P=0.032)$. Similar associations were observed after 3 months and after 6 months when we added smoking status, drinking habit and exercise habit as control variables $(r=0.070, P=0.432$ and $r=-0.203, P=0.020$, respectively).

Table 3 Correlations between changes in Angptl2 (left) and adiponectin (right) levels and changes in adiposity, blood pressure and laboratory data at 3 and 6 months after beginning the intervention relative to baseline data, which was estimated before the intervention began

\begin{tabular}{|c|c|c|c|c|c|c|c|c|}
\hline \multirow[t]{3}{*}{ From baseline } & \multicolumn{4}{|c|}{$\Delta$ Angpt/2 } & \multicolumn{4}{|c|}{$\Delta$ Adiponectin } \\
\hline & \multicolumn{2}{|c|}{ To 3 months } & \multicolumn{2}{|c|}{ To 6 months } & \multicolumn{2}{|c|}{ To 3 months } & \multicolumn{2}{|c|}{ To 6 months } \\
\hline & $\mathrm{r}$ & $\mathrm{P}$ & $r$ & $\mathrm{P}$ & $r$ & $\mathrm{P}$ & $r$ & $P$ \\
\hline \multicolumn{9}{|l|}{$\Delta$ Adiposity } \\
\hline$\Delta \mathrm{BMI}$ & 0.370 & $<0.001$ & 0.362 & $<0.001$ & -0.190 & 0.028 & -0.190 & $<0.001$ \\
\hline$\Delta$ Abdominal circumference & 0.265 & 0.002 & 0.311 & $<0.001$ & -0.017 & 0.843 & -0.017 & 0.003 \\
\hline$\Delta$ Fat mass & 0.254 & 0.003 & 0.353 & $<0.001$ & -0.209 & 0.015 & -0.209 & $<0.001$ \\
\hline \multicolumn{9}{|l|}{$\Delta$ Clinical and laboratory data } \\
\hline$\triangle \mathrm{SBP}$ & 0.078 & 0.372 & 0.021 & 0.812 & 0.071 & 0.415 & -0.066 & 0.451 \\
\hline$\triangle \mathrm{DBP}$ & 0.165 & 0.057 & 0.119 & 0.172 & 0.106 & 0.226 & 0.044 & 0.612 \\
\hline$\Delta \mathrm{TG}$ & 0.360 & $<0.001$ & 0.194 & 0.025 & 0.102 & 0.241 & 0.080 & 0.359 \\
\hline$\Delta \mathrm{HDL}-\mathrm{C}$ & -0.055 & 0.526 & -0.196 & 0.024 & 0.203 & 0.019 & 0.369 & $<0.001$ \\
\hline$\Delta \mathrm{LDL}-\mathrm{C}$ & -0.114 & 0.193 & -0.100 & 0.250 & 0.179 & 0.039 & 0.145 & 0.096 \\
\hline$\triangle \mathrm{FPG}$ & 0.021 & 0.813 & 0.157 & 0.072 & -0.132 & 0.130 & -0.273 & 0.001 \\
\hline$\Delta \mathrm{HbA} 1 \mathrm{c}$ & 0.082 & 0.350 & 0.107 & 0.221 & 0.134 & 0.123 & 0.045 & 0.610 \\
\hline$\Delta$ Insulin & -0.086 & 0.324 & -0.037 & 0.676 & 0.040 & 0.649 & 0.081 & 0.355 \\
\hline$\Delta$ HOMA-IR & -0.109 & 0.213 & -0.044 & 0.615 & 0.024 & 0.782 & 0.070 & 0.425 \\
\hline$\Delta \mathrm{AST}$ & 0.272 & 0.002 & 0.195 & 0.024 & 0.110 & 0.208 & -0.066 & 0.454 \\
\hline$\Delta \mathrm{ALT}$ & 0.216 & 0.012 & 0.161 & 0.064 & 0.118 & 0.176 & -0.032 & 0.718 \\
\hline$\Delta$ Log Hs-CRP & 0.241 & 0.005 & 0.257 & 0.003 & -0.062 & 0.480 & -0.021 & 0.809 \\
\hline$\Delta$ Angptl2 & - & - & - & - & 0.068 & 0.436 & -0.186 & 0.032 \\
\hline$\Delta$ Adiponectin & 0.068 & 0.436 & -0.186 & 0.032 & - & - & - & - \\
\hline
\end{tabular}

Abbreviations: Angptl2, angiopoietin-like protein 2; ALT, alanine aminotransferase; AST, aspartate aminotransferase; BMI, body mass index; DBP, diastolic blood pressure; FPG, fasting plasma glucose; HbA1c, hemoglobin A1c; HOMA-IR, homeostasis model assessment of the insulin resistance index; Hs-CRP, high sensitive C-reactive protein; HDL-C, high-density lipoprotein cholesterol; LDL-C, low-density lipoprotein cholesterol; SBP, systolic blood pressure; TG, triglyceride. Analysis was performed and adjusted by age (for adiposity) and BMI (for clinical and laboratory data). $r$ and $P$ indicate correlation coefficients and $P$-values, respectively. 
Table 4 Multiple linear regression analysis to examine changes in clinical data mediated by the intervention

\begin{tabular}{|c|c|c|c|c|c|c|c|c|c|c|c|c|}
\hline \multirow[t]{3}{*}{ Target variable } & \multicolumn{12}{|c|}{ Explanatory variable } \\
\hline & \multicolumn{2}{|c|}{$\triangle B M I$} & \multicolumn{2}{|c|}{$\triangle$ Abdominal Circumference } & \multicolumn{2}{|c|}{$\Delta$ Fat mass } & \multicolumn{2}{|c|}{$\triangle \log H s-C R P$} & \multicolumn{2}{|c|}{$\Delta$ Angpt 12} & \multicolumn{2}{|c|}{ AAdiponectin } \\
\hline & $\beta$ & $\mathrm{P}$ & $\beta$ & $P$ & $\beta$ & $\mathrm{P}$ & $\beta$ & $\mathrm{P}$ & $\beta$ & $\mathrm{P}$ & $\beta$ & $P$ \\
\hline \multicolumn{13}{|c|}{ From baseline to 3 months } \\
\hline$\triangle \mathrm{DBP}$ & 0.023 & 0.872 & 0.151 & 0.223 & -0.127 & 0.234 & -0.079 & 0.379 & 0.197 & 0.04 & 0.056 & 0.53 \\
\hline$\Delta \mathrm{TG}$ & -0.103 & 0.431 & 0.07 & 0.54 & 0.17 & 0.084 & -0.195 & 0.019 & 0.42 & $<0.001$ & 0.077 & 0.348 \\
\hline$\Delta \mathrm{HDL}-\mathrm{C}$ & -0.167 & 0.238 & 0.075 & 0.542 & 0.073 & 0.492 & -0.041 & 0.649 & -0.066 & 0.486 & 0.208 & 0.02 \\
\hline$\Delta \mathrm{LDL}-\mathrm{C}$ & 0.531 & $<0.001$ & -0.175 & 0.135 & 0.045 & 0.655 & 0.032 & 0.701 & -0.141 & 0.117 & 0.189 & 0.025 \\
\hline$\Delta \mathrm{AST}$ & 0.044 & 0.74 & 0.085 & 0.464 & 0.178 & 0.075 & -0.009 & 0.919 & 0.268 & 0.003 & 0.111 & 0.183 \\
\hline$\Delta \mathrm{ALT}$ & 0.116 & 0.384 & -0.039 & 0.739 & 0.241 & 0.017 & -0.034 & 0.682 & 0.213 & 0.019 & 0.144 & 0.086 \\
\hline \multicolumn{13}{|c|}{ From baseline to 6 months } \\
\hline$\Delta \mathrm{TG}$ & -0.079 & 0.642 & 0.168 & 0.272 & 0.088 & 0.455 & -0.015 & 0.868 & 0.221 & 0.023 & 0.129 & 0.177 \\
\hline$\Delta \mathrm{HDL}-\mathrm{C}$ & -0.185 & 0.24 & 0.148 & 0.294 & -0.048 & 0.659 & -0.047 & 0.566 & -0.123 & 0.166 & 0.338 & $<0.001$ \\
\hline$\Delta \mathrm{FPG}$ & 0.176 & 0.277 & -0.104 & 0.474 & 0.103 & 0.358 & 0.028 & 0.744 & 0.09 & 0.326 & -0.257 & 0.005 \\
\hline
\end{tabular}

Abbreviations: Angptl2, angiopoietin-like protein 2; ALT, alanine aminotransferase; AST, aspartate aminotransferase; BMI, body mass index; DBP, diastolic blood pressure; FPG, fasting plasma glucose; HbA1c, hemoglobin A1c; HOMA-IR, homeostasis model assessment of the insulin resistance index; Hs-CRP, high sensitive C-reactive protein; HDL-C, high-density lipoprotein cholesterol; LDL-C, low-density lipoprotein cholesterol; SBP, systolic blood pressure; TG, triglyceride. Target variables: changes in SBP, DBP, TG, HDL-C, LDL-C, FPG, HbA1c, insulin, HOMA-IR, AST and ALT levels from intervention onset to 3 and 6 months. Explanatory variables: changes in BMI, abdominal circumference, fat mass, log hs-CRP, Angptl2 and adiponectin levels at 3- and 6-month time points.

We next performed multiple linear regression analysis to examine which changes contributed to improvement of clinical data. As shown in Table 4, changes in serum Angptl2 concentrations were positively correlated with diastolic blood pressure, TG, AST and ALT values, whereas changes in serum adiponectin concentrations were positively correlated with HDL-C values at 3 months. At the end of the program (6 months), changes in TG were positively correlated with serum Angptl2 concentrations, whereas changes in HDL-C were positively correlated with serum adiponectin concentrations, while FPG changes were inversely correlated with adiponectin levels.

Weight reduction rates and changes in laboratory parameters At 3 and 6 months after the beginning of the intervention, subjects were grouped by the degree of weight reduction rate into 5 groups: a weight gain group (3 months; $n=19$, 6 months; $n=23$ ), the 0 to $<2 \%$ weight reduction group, which was designated as the unchanged control group for the following analysis ( 3 months; $n=35$, 6 months; $n=32$ ), the 2 to $<4 \%$ weight reduction group ( 3 months; $n=41$, 6 months; $n=26$ ), the 4 to $<6 \%$ weight reduction group (3 months; $n=20,6$ months; $n=17$ and the $6 \%$ or more weight reduction group (3 months; $n=20,6$ months; $n=37)$.

At the 3-month time point, one-way analysis revealed significant differences in alteration in LDL-C, FPG, AST and ALT among the 5 groups (Figure 2a). Multiple comparison analysis revealed significant decreases in LDL-C in the $6 \%$ or more weight reduction group and significant decreases in ALT in the 4 to $<6 \%$ weight reduction group compared with the unchanged control group. At the 6-month time point, one-way analysis revealed significant differences in alteration of HDL-C, LDL-C, FPG, HbA1c, AST and ALT among the 5 groups (Figure 2b). Multiple comparison analysis showed significant decreases in LDL-C in the $6 \%$ or more weight reduction group and decreases in AST and ALT in the $4-<6 \%$ group and $6 \%$ or more compared with the unchanged control group.

One-way analysis also revealed significant differences in alteration of serum Angptl2 levels among the 5 groups at both 3- and 6-month time points (Figures $2 \mathrm{c}$ and d), whereas significant alterations in serum adiponectin concentration were seen only at the 6 month time point. Serum Angptl2 concentrations tended to decrease with increased percentage of weight reduction, and the $6 \%$ or more weight reduction group showed a significant decrease relative to the unchanged control group at both the 3- and 6-month time points (Figures 2c and d). By contrast, serum adiponectin concentrations tended to increase with increased percentage of weight reduction, and the $6 \%$ or more weight reduction group showed a significant increase compared with the unchanged control group at 6 months after the beginning of the intervention, a change that was not observed at the 3-month time point (Figures 2c and d).

\section{Discussion}

We conducted a 3-month lifestyle intervention for men who were overweight and observed maintenance of weight reduction for another 3 months thereafter. Significant weight reduction was obtained, improved lipid and glucose metabolism and lowered plasma liver enzymes were observed immediately after the end of the 3-month lifestyle intervention. Moreover, improvement was maintained until the 6-month time point. Notably, decreased serum Angptl2 
levels were seen immediately after the end of 3-month lifestyle intervention and continued for the entire 6-month period. This finding was significant in the $6 \%$ or more weight reduction group. By contrast, an expected increase in serum adiponectin levels was observed only in subjects with $\geqslant 6 \%$ weight reduction at the 6-month time point.
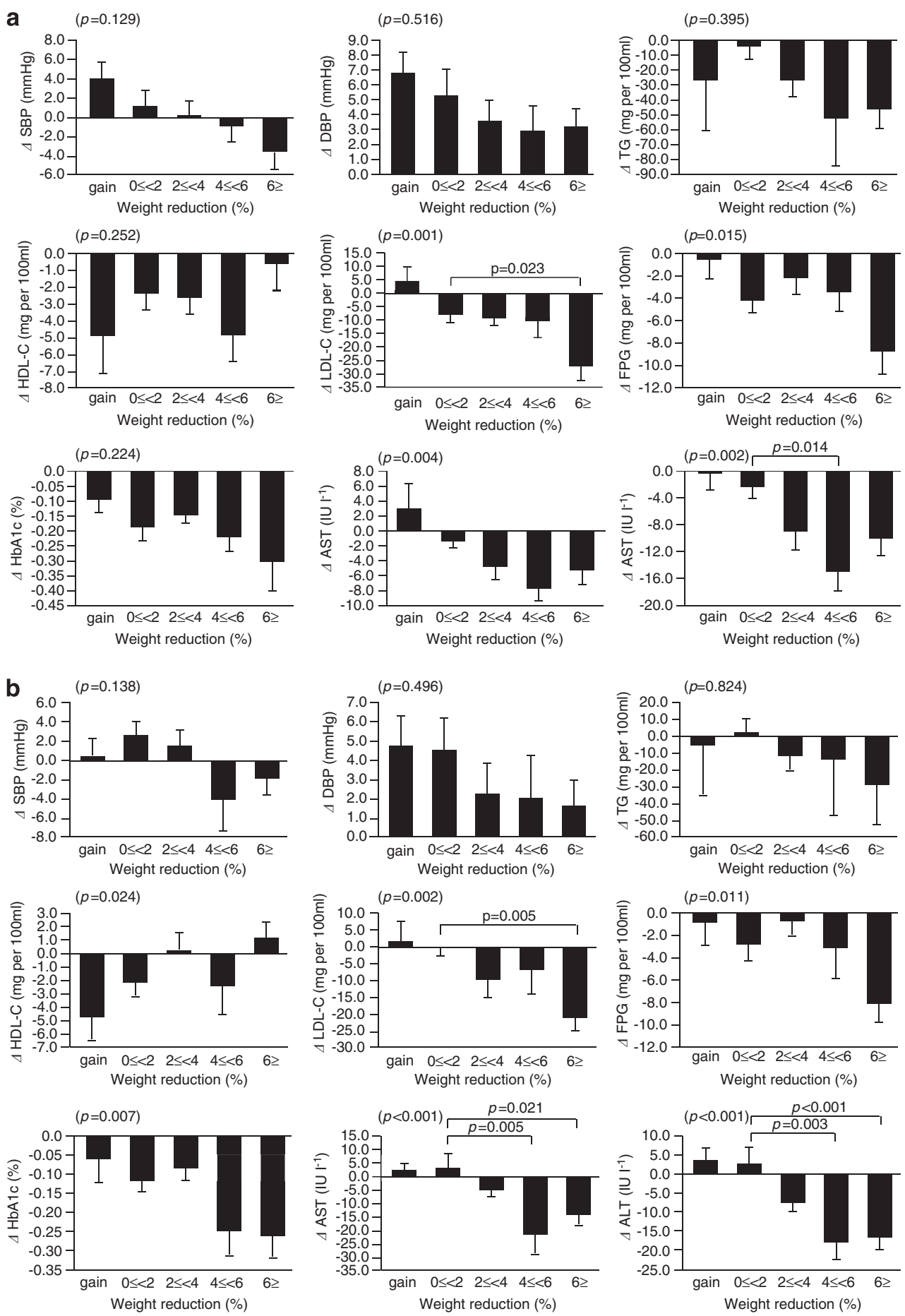

Figure 2 Changes in BP, laboratory parameters related to lipid and glucose metabolism and liver function, and serum Angptl2 and adiponectin levels in each weight reduction group. (a, b) Changes are evaluated at 3 (a) and 6 (b) months after beginning the intervention. (c, d) Changes in serum Angptl 2 and adiponectin at 3 (c) and 6 (d) months after beginning the intervention. Vertical axes indicate changes in laboratory data, and the horizontal axes indicate percent weight reduction. Data were analyzed by one-way analysis of variance analysis of variance and compared between groups by multiple comparisons using the Bonferroni method. The Kruskal-Wallis test was used to evaluate changes in DBP and HbA1c at the 3-month point (a). Data are presented as means \pm s.e.m. 

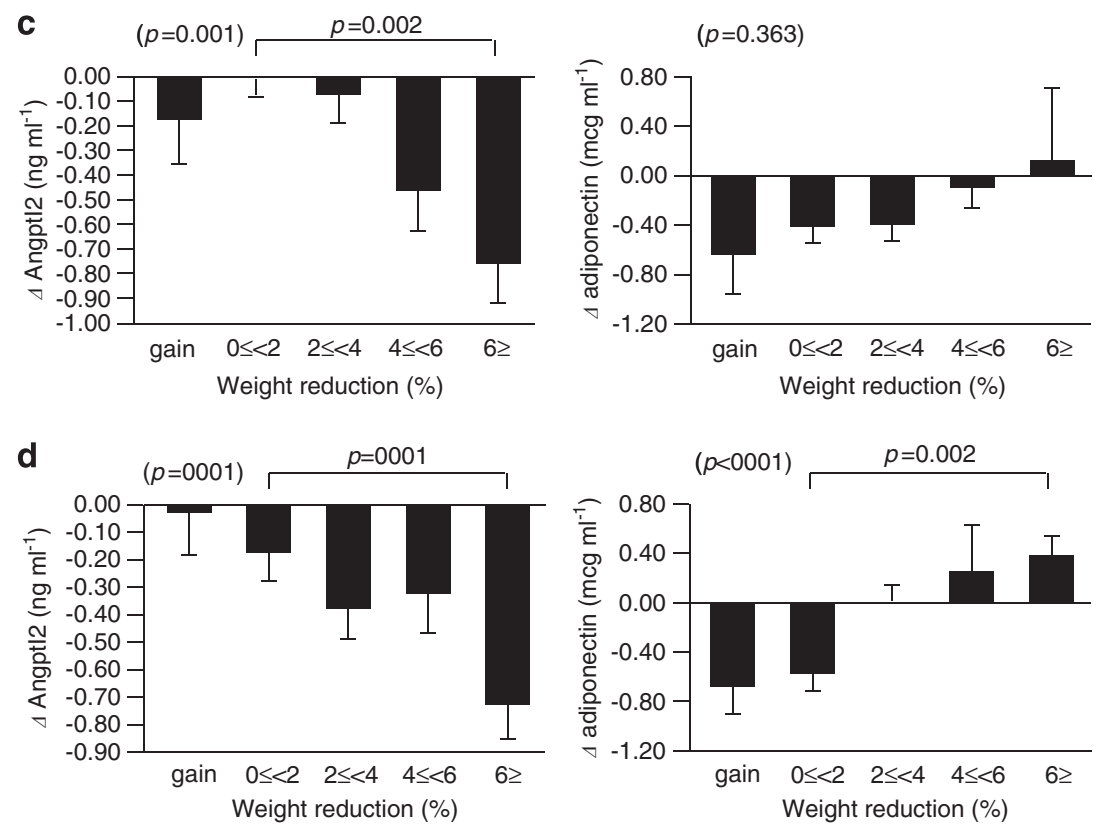

Figure 2 Continued.

In previously reported intervention studies, such as the Diabetes Prevention Program, ${ }^{28}$ the Finnish Diabetes Prevention Study ${ }^{29}$ and the Malmo feasibility study, ${ }^{30}$ longer 6- or 12-month interventions supported on a one-to-one basis by case managers were undertaken. By comparison, our intervention was mild, because only one or a few case workers were available to the subjects for 3 months. However, it is noteworthy that our intervention resulted in significant weight reduction and moreover, improvement was maintained for another 3 months after the end of the first 3-month intervention.

Many previous studies have shown that circulating adiponectin concentrations are inversely correlated with adiposity and BMI. ${ }^{6-9}$ Decreased adiponectin was found in cases of visceral fat accumulation, and, conversely, weight reduction promoted adiponectin increases. ${ }^{6-9}$ Here, significantly increased serum adiponectin levels were not detected until 6 months after the intervention was started, whereas loss of body weight and adiposity as estimated by BMI, abdominal circumference, percent fat and fat mass were detected at 3 months after the beginning of intervention. These facts might mean adiponectin responds to weight reduction slowly, so early changes in labolatroy data might not be induced by adiponectin.

Recently, the multimeric adiponectin is considered the active form and is better correlated with metabolic parameters. Bobbert et al. ${ }^{7}$ reported that weight reduction brought increased quantities of high molecular weight isoforms of adiponectin, but total adiponectin showed no change. On the other hand, some studies have shown increased total adiponectin by lifestyle intervention. ${ }^{6,8}$
In this study, we measured total adiponectin, so further studies will be needed for the multimeric adiponectin.

When we examined the association between weight reduction percentage and adiponectin levels, adiponectin began to increase in the $>6 \%$ weight reduction group after 3 months and in the $>4 \%$ weight reduction groups after 6 months; however, significant adiponectin increases were restricted to the $>6 \%$ weight reduction groups after 6 months. Thus, greater weight reduction over longer followup periods are required to detect increases in circulating adiponectin after an intervention is initiated. Nonetheless, changes in HDL-C were positively correlated with serum adiponectin concentrations, and changes in FPG and adiposity as estimated by BMI, abdominal circumference and fat mass showed an inverse relationship with adiponectin levels. Overall, our observations suggest that adiponectin might not be a highly sensitive marker of improved metabolism at early time points when substantial weight loss is not yet apparent, but rather may be a good marker of metabolic improvement in the late phase based on normalization of adipocytes following significant weight loss. This idea is consistent with the idea that adiponectin is produced from only adipocytes, and its production from enlarged and/or inflammatory adipocytes seen in obesity is significantly decreased. ${ }^{6-9}$

Increased Angptl2 levels owing to visceral fat accumulation cause chronic inflammation and subsequent metabolic disturbance. ${ }^{20-22}$ We found that Angptl2 levels tended to decrease immediately in subjects showing $2 \%$ or more weight reduction and continued until 3 months after the intervention. Angptl2 gradually decreased with increased 
weight reduction, and those decreases in the $6 \%$ or more weight reduction group were significant compared with the unchanged control group, indicative of an early effect of weight reduction on improved metabolism. We speculate that differences in changes of circulating levels of Angptl2 and adiponectin after the intervention are due to the types of cells expressing each factor: Angptl2 is produced by adipocytes and other cell types, such as vascular endothelia cells and monocyte/macrophages, while adiponectin expression is restricted to adipocytes. Angptl2 level changes showed a significant positive correlation with changes in adiposity and levels of hs-CRP, TG, AST and ALT and a significant inverse correlation with HDL-C and adiponectin levels. By contrast, adiponectin levels showed a significant positive correlation with changes in HDL-C and a significant inverse correlation with the changes in FPG, adiposity and Angpt12. Interestingly, Angptl2 and adiponectin levels were inversely correlated. It is noteworthy that changes in Angptl2 levels are closely associated with changes in inflammation, TG metabolism and ALT, whereas changes in adiponectin are associated with glucose metabolism.

There are several limitations to this study. We used BIA method to determine body fat percentage. Some studies showed a good relationship between BIA and dual-energy $X$-ray absorptiometory, ${ }^{31}$ whereas others indicate that the BIA method lacked accuracy. ${ }^{32}$ Further studies are required to evaluate Angpt12 as an appropriate marker of amelioration of obesity and its-related metabolic disturbances. In particular, it is important to determine whether changes of Angptl2 observed here apply over longer follow-up periods and to a wider population of subjects, such as females, or individuals with severe obesity or with metabolic disease.

In conclusion, we showed that a 3-month lifestyle intervention induced weight reduction and improved glucose and lipid metabolism, changes that continued for 3 months thereafter. Our findings indicate that decreased Angptl2 levels are a good indicator of reduced visceral fat and metabolic improvement at early stages of lifestyle intervention. Thus, Angptl2 reflects adiposity and might be a key protein to regulate inflammation and TG metabolism, whereas adiponectin levels could reflect improved glucose and HDL-C metabolism.

\section{Conflict of interest}

The authors declare no conflict of interest.

\section{Acknowledgements}

We express our sincere appreciation to Mr Yoshiki Aratani, Ms Kaori Itakura, Ms Chiaki Ono, Mr Takuya Ozeki, Mr Masaki Wada and other members of the Aichi Health Plaza Comprehensive Health Science Center who contributed to this study. Dr Kazuyo Tsushita was funded by the Health Labour Sciences Research Grant and Suzuken memorial foundation. Dr Yuichi Oike was supported by Grants-in-Aid for Scientific Research on Priority Areas from the Ministry of Education, Culture, Sports, Science and Technology of Japan (No. 21390245), and by grants from the Takeda Science Foundation, and the Tokyo Biochemical Research Foundation.

\section{References}

1 Lee IM, Manson JE, Hennekens CH, Paffenbarger Jr RS. Body weight and mortality. A 27-year follow-up of middle-aged men. JAMA 1993; 270: 2823-2828.

2 Reaven GM. Syndrome X: 6 years later. I Intern Med Suppl 1994; 736: 13-22.

3 Stevens J, Cai J, Pamuk ER, Williamson DF, Thun MJ, Wood JL. The effect of age on the association between body-mass index and mortality. $N$ Engl J Med 1998; 338: 1-7.

4 Eckel RH, Grundy SM, Zimmet PZ. The metabolic syndrome. Lancet 2005; 365: 1415-1428.

5 Mokdad AH, Ford ES, Bowman BA, Dietz WH, Vinicor F, Bales VS et al. Prevalence of obesity, diabetes, and obesity-related health risk factors, 2001. JAMA 2003; 289: 76-79.

6 Esposito K, Pontillo A, Di Palo C, Giugliano G, Masella M, Marfella $\mathrm{R}$ et al. Effect of weight loss and lifestyle changes on vascular inflammatory markers in obese women: a randomized trial. JAMA 2003; 289: 1799-1804.

7 Bobbert T, Rochlitz H, Wegewitz U et al. Changes of adiponectin oligomer composition by moderate weight reduction. Diabetes 2005; 54: 2712-2719.

8 Mather KJ, Funahashi T, Matsuzawa Y, Edelstein S, Bray GA, Kahn SE et al. Adiponectin, change in adiponectin, and progression to diabetes in the Diabetes Prevention Program. Diabetes 2008; 57: 980-986.

9 Madsen EL, Rissanen A, Bruun JM, Skogstrand K, Tonstad S, Hougaard DM et al. Weight loss larger than 10\% is needed for general improvement of levels of circulating adiponectin and markers of inflammation in obese subjects: a 3-year weight loss study. Eur J Endocrinol 2008; 158: 179-187.

10 Engström G, Hedblad B, Stavenow L, Lind P, Janzon L, Lindgärde F. Inflammation-sensitive plasma proteins are associated with future weight gain. Diabetes 2003; 52: 2097-2101.

11 Festa A, D'Agostino Jr R, Howard G, Mykkänen L, Tracy RP, Haffner SM. Chronic subclinical inflammation as part of the insulin resistance syndrome: the Insulin Resistance Atherosclerosis Study (IRAS). Circulation 2000; 102: 42-47.

12 Festa A, D'Agostino Jr R, Tracy RP, Haffner SM. Insulin Resistance Atherosclerosis Study. Elevated levels of acute-phase proteins and plasminogen activator inhibitor-1 predict the development of type 2 diabetes: the insulin resistance atherosclerosis study. Diabetes 2002; 51: 1131-1137.

13 Koenig W, Sund M, Fröhlich M, Fischer HG, Löwel H, Döring A et al. C-Reactive protein, a sensitive marker of inflammation, predicts future risk of coronary heart disease in initially healthy middle-aged men: results from the MONICA (Monitoring Trends and Determinants in Cardiovascular Disease) Augsburg Cohort Study, 1984 to 1992. Circulation 1999; 99: 237-242.

14 Retterstol L, Eikvar L, Bohn M, Bakken A, Erikssen J, Berg K. C-reactive protein predicts death in patients with previous premature myocardial infarction-a 10 year follow-up study. Atherosclerosis 2002; 160: 433-440.

15 Jousilahti P, Salomaa V, Rasi V, Vahtera E, Palosuo T. The association of c-reactive protein, serum amyloid a and fibrinogen 
with prevalent coronary heart disease-baseline findings of the PAIS project. Atherosclerosis 2001; 156: 451-456.

16 Kopp HP, Kopp CW, Festa A, Krzyzanowska K, Kriwanek S, Minar E et al. Impact of weight loss on inflammatory proteins and their association with the insulin resistance syndrome in morbidly obese patients. Arterioscler Thromb Vasc Biol 2003; 23: 1042-1047.

17 Ryan AS, Nicklas BJ. Reductions in plasma cytokine levels with weight loss improve insulin sensitivity in overweight and obese postmenopausal women. Diabetes Care 2004; 27: 1699-1705.

18 Heilbronn LK, Noakes M, Clifton PM. Energy restriction and weight loss on very-low-fat diets reduce C-reactive protein concentrations in obese, healthy women. Arterioscler Thromb Vasc Biol 2001; 21: 968-970.

19 Yatsuya H, Jeffery RW, Langer SL, Mitchell N, Flood AP, Welsh EM et al. Changes in C-reactive protein during weight loss and the association with changes in anthropometric variables in men and women: LIFE Study. Int J Obes (Lond) 2010; 35: 684-691.

20 Tabata M, Kadomatsu T, Fukuhara S, Miyata K, Ito Y, Endo M et al. Angiopoietin-like protein 2 promotes chronic adipose tissue inflammation and obesity-related systemic insulin resistance. Cell Metab 2009; 10: 178-188.

21 Oike Y, Tabata M. Angiopoietin-like proteins-potential therapeutic targets for metabolic syndrome and cardiovascular disease. Circ J 2009; 73: 2192-2197.

22 Ouchi N, Parker JL, Lugus JJ, Walsh K. Adipokines in inflammation and metabolic disease. Nat Rev Immunol 2011; 11: 85-97.

23 The Examination Committee of Criteria for 'Obesity Disease' in Japan, Japan Society for the Study of Obesity. New criteria for 'obesity disease'. Japan Circ J 2002; 66: 987-992.

24 Seino Y, Nanjo K, Tajima N, Kadowaki T, Kashiwagi A, Araki E et al. Report of the Committee on the classification and diagnostic criteria of diabetes mellitus. Diabetol Int 2010; 1: 2-20.
25 Haffner SM, Miettinen H, Stern MP. The homeostasis model in the San Antonio Heart Study. Diabetes Care 1997; 20: 1087-1092.

26 Wallace TM, Levy JC, Matthews DR. Use and abuse of HOMA modeling. Diabetes Care 2004; 27: 1487-1495.

27 Okada T, Tsukano H, Endo M, Tabata M, Miyata K, Kadomatsu T et al. Synoviocyte-derived Angiopoietin-like protein 2 contributes to synovial chronic inflammation in rheumatoid arthritis. Am J Pathol 2010; 176: 2309-2319.

28 Knowler WC, Barrett-Connor E, Fowler SE et al. Diabetes Prevention Program Research Group. Reduction in the incidence of type 2 diabetes with lifestyle intervention or metformin. $N$ Engl $J$ Med 2002; 346: 393-403.

29 Tuomilehto J, Lindstrom J, Eriksson JG et al. Prevention of type 2 diabetes mellitus by changes in lifestyle among subjects with impaired glucose tolerance. N Engl J Med 2001; 344: 1343-1350.

30 Eriksson KF, Lindgärde F. Prevention of type 2 (non-insulindependent) diabetes mellitus by diet and physical exercise. The 6-year Malmö feasibility study. Diabetologia 1991; 34: 891-898.

31 Bolanowski M, Nilsson RE. Assessment of human body composition using dual-energy $\mathrm{x}$-ray absorptiometry and bioelectrical impedance analysis. Med Sci Monit 2001; 5: 1029-1033.

32 Pateyjohns IR, Brinkworth GD, Buckley JD, Noakes M, Clifton PM. Comparison of three bioelectrical impedance methods with DXA in overweight and obese men. Obesity 2006; 14: 2064-2070.

This work is licensed under the Creative Commons Attribution-NonCommercial-No Derivative Works 3.0 Unported License. To view a copy of this license, visit http://creativecommons.org/ licenses/by-nc-nd/3.0/ 